\title{
Evaluation de la prevalence, de la typologie et des complications liees a l'excision chez les patientes frequentant les formations sanitaires du Bazega
}

Direction de la Santé et de la Famille (DSF)

University of Ouagadougou Demographic Research Unit (UERD)

Mwangaza

Population Council

Follow this and additional works at: https://knowledgecommons.popcouncil.org/departments_sbsr-rh How does access to this work benefit you? Let us know!

\section{Recommended Citation}

"Evaluation de la prevalence, de la typologie et des complications liees a l'excision chez les patientes frequentant les formations sanitaires du Bazega [Burkina Faso]," Rapport Final du Projet de Recherche Operationnelle et d'Assistance Technique en Afrique II. Ouagadougou: Population Council, Min de la Santé, Direction de la Santé de la Famille, Unité d'Enseignement Recherche Démographie, and Mwangaza, 1998. 


\section{Laboratoire de SANTE Communautaire, BAZEGA}

\section{EVALUATION DE LA PREVALENCE, DE LA TYPOLOGIE ET DES COMPLICATIONS LIEES A L'EXCISION CHEZ LES PATIENTES FREQUENTANT LES FORMATIONS SANITAIRES DU BAZEGA}

Série Documentaire $N^{\circ} 21$

MINISTERE DE LA SANTE

Direction de la Santé de la Famille
Unité d'Enseignement et de Recherche en Démographie
Mwangaza

Population Council

Africa OR/TA II

Ouagadougou

Burkina Faso

Novembre 1998 


\section{TABLE DES MATIERES}

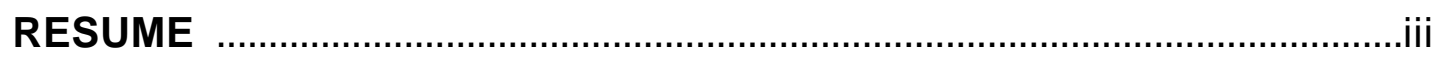

EXECUTIVE SUMMARY _...................................................................

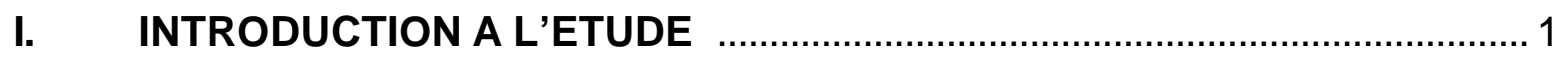

1.1. Contexte et identification du problème ................................................. 1

1.2. Justification de l'étude ........................................................................ 2

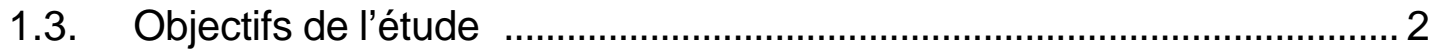

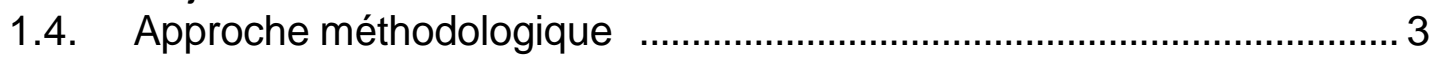

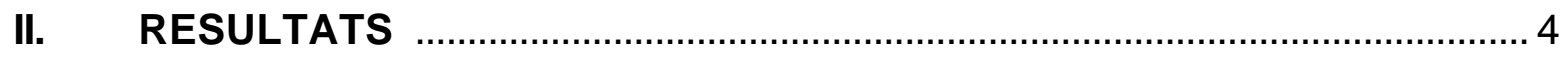

2.1. Motif de consultation ...................................................................... 4

2.2. Les caractéristiques socio-démographiques ......................................... 4

2.3. Statut obstétrical ............................................................................... 5

2.4. Expérience des clientes en matière d'excision ....................................... 5

2.5. Complications observées ................................................................ 7

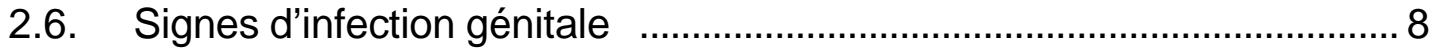

2.7. Difficultés à l'accouchement ................................................................. 8

2.8. Perspectives et attitudes des femmes en matière d'excision ................. 9

III. DISSEMINATION ET UTILISATION .........................................................

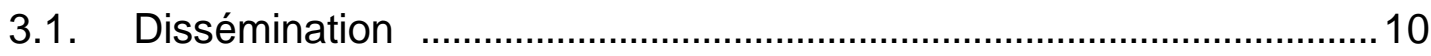

3.2. Utilisation des résultats ..................................................................10

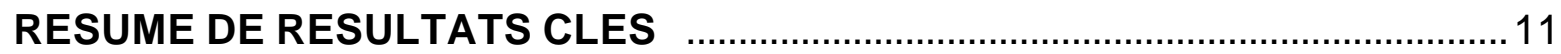

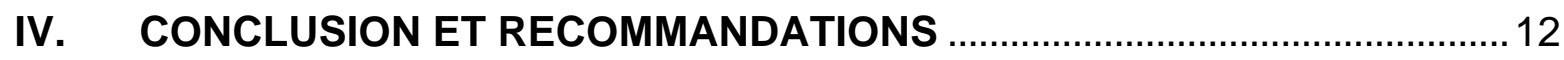

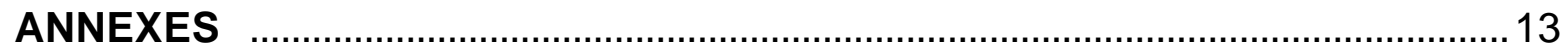

Annexe 1. Echantillonage des formations sanitaires et des enquêtées par zone 
- CIPD

- CNLPE

- CPN

- DSF

- EDS

- FS

- IEC

- LSC

- MGF

- MST

- OMS

- ONG

- $P F$

- RO/AT II Phase II

- SIDA
Conférence internationale sur la population et le développement

Comité national de lutte contre la pratique de l'excision

Consultation prénatale

Direction de la santé de la famille

Enquête Démographique et de Santé

Formation sanitaire

Information, éducation, communication

Laboratoire de santé communautaire

Mutilation génitale féminine

Maladie sexuellement transmissible

Organisation mondiale de la santé

Organisation non-gouvernementale

Planification familiale

Recherche Opérationnelle et Assistance Technique en Afrique

Syndrome immuno-déficitaire acquis 


\section{RESUME}

\section{Introduction}

Cette étude est une contribution à la lutte contre la pratique de l'excision au Burkina Faso. Des études ont montré que l'excision est prévalente mais très peu de recherches ont porté sur la typologie et les complications liées à l'excision. Cette étude vise donc à l'évaluation de la typologie et des complications liées à l'excision chez les clientes fréquentant les formations sanitaires du Bazèga et du Zoundwéogo et ce, dans le cadre des activités du Laboratoire de Santé Communautaire (LSC).

\section{Objectifs}

Cette étude avait deux objectifs principaux : i) déterminer la prévalence de la typologie de l'excision pratiquée dans la zone du LSC ; ii) identifier les complications liées à l'excision.

\section{Méthodologie}

II s'agit d'une étude de nature quantitative qui s'est déroulée dans la zone d'intervention du LSC. Elle a concerné un échantillon fortuit de 1920 clientes recrutées dans 21 formations sanitaires sur une période trois (3) mois, de mai à juillet 1998. Toute cliente venant dans l'une des 21 formations sanitaires (FS), dont le motif de consultation nécessitait un examen pelvien et qui donnait son consentement verbal était incluse dans l'étude. Quarante deux (42) prestataires ont été formés à l'identification des types d'excision et des complications et à remplir le guide confectionné à cet effet.

\section{Résultats clés}

Un total de 1920 clientes ont été interviewées durant la période de l'étude. Plus de la moitié d'entre elles étaient venues pour des consultations prénatales (57\%) et $12 \%$ pour planification familiale. Elles avaient un âge compris entre 15 et 55 ans, mais dans l'ensemble, elles étaient relativement jeunes, mariées, d'ethnie Mossi dans la majorité (l'étude se déroule dans une zone Mossi). Les religions, musulmane et catholique étaient les principales religions rencontrées. La majorité (77\%) n'avait aucun niveau d'instruction.

Parmi les 1920 femmes, 93\% étaient excisées contre 7\% non excisées.

Trois types d'excision, selon la classification de l'OMS ont été observés ; le type I est observé dans plus de la moitié des cas, $56 \%$, le type II dans 39\% et le type III dans $5 \%$.

L'âge à l'excision est variable mais tend à baisser. La moyenne est de huit (8) ans dans cette étude. La quasi totalité des femmes excisées le sont avant l'âge de 18 ans.

Plusieurs instruments sont utilisés pour pratiquer l'opération, à savoir des lames, des couteaux, des fibres de tiges de mil ou des pierres taillées.

Après l'excision, les soins sont en général assurés par les parents dans plus de la moitié des cas tandis que les exciseuses interviennent dans $1 / 3$ des cas. Des produits modernes et traditionnels sont utilisés isolément en combinaison pour les soins des 
plaies. II s'agit entre autres de la pommade pénicilline, de l'alcool avec de la bouse de vache, des racines ou du beurre de karité.

Parmi les femmes excisées, il a été observé lors de l'examen pelvien des complications liées à l'excision chez $14 \%$ d'entre elles. Ces complications sont surtout des chéloïdes observées chez $62 \%$ des clientes, des sténoses dans $20 \%$ des cas et des obstructions vaginales. Statistiquement parmi les femmes excisées, celles relevant du type III étaient les plus propices à en avoir. Un lien a aussi été établi entre l'excision et la probabilité qu'une difficulté à l'accouchement soit reportée.

L'étude a voulu connaître les perspectives des clientes en matière d'excision; la situation semble favorable dans la mesure où dans l'ensemble les clientes ne sont pas favorables à l'excision de leur fille à l'avenir de même que celles qui ne sont pas excisées ne souhaitent pas l'être dans l'avenir. Ces constatations varient en fonction du niveau d'éducation et du statut d'excision de la cliente. Mais cette situation doit être interprétée en prenant en compte le contexte du Burkina où l'excision est interdite par la loi.

\section{Conclusion et recommandations}

Cette étude, bien que limitée à une zone socio-culturelle du Burkina, fournit des indications et des données pouvant contribuer à la lutte contre la pratique de l'excision.

Les résultats ont été diffusés au niveau national. Conformément à la philosophie du LSC, ils devraient être disséminés auprès de la communauté et de tous les acteurs travaillant sur le terrain.

Les résultats devraient être utilisés comme argumentaires pendant les séances d'information, d'éducation et de communication tant au niveau communautaire qu'au niveau national.

Etant donné que les prestataires ont reçu une formation, il serait utile et pertinent d'intégrer la lutte contre l'excision dans les prestations courantes des formations sanitaires. 


\section{EXECUTIVE SUMMARY}

\section{Introduction}

FGC is prevalent in Burkina Faso, where an estimated $70 \%$ of women have undergone this procedure. Little information exists on the types of FGC found in Burkina Faso, or on the resulting physical complications. This study was thus undertaken to evaluate the typology of FGC and visible effects among clients attending health clinics in the rural provinces of Bazega and Zoundweogo. The study was undertaken as part of the activities of the field research station in Bazega, or the Laboratoire de Santé Communautaire (LSC). The results are expected to add to the literature on female genital cutting (FGC), helping to inform those working to end the practice.

\section{Objectives}

There were two primary objectives: i) to determine the prevalence of the types of FGC, as defined by the WHO, found in the LSC catchment area; ii) to identify the complications resulting from FGC, and any associations between complications and the specific type of FGC experienced.

\section{Methodology}

This quantitative study took place at the service delivery points (SDPs) of a larger fourcelled operations research project undertaken by the LSC. The sample consists of 1,920 clients from the 21 SDPs found in the LSC catchment area, over a three-month period. This group includes all of the women who had a pelvic exam. Only women giving verbal consent were included. Forty-two service providers were trained to identify the different types of FGC and observable complications.

\section{Key findings}

The majority of clients receiving a pelvic exam came in for a prenatal consultation (57\%), followed by $12 \%$ for family planning and $12 \%$ for delivery. The age of these clients ranged from 15 to 55, but the majority were relatively young (mean age of 27), married, and from the Mossi ethnic group. Catholicism and Islam were the major religions found in the sample. The majority of clients had no formal education (77\%).

Among the 1,920 women interviewed, 93\% had undergone genital cutting, 7\% had not. Three of the four types of FGC were found: type I or clitoridectomy was the most common (56\% of cases), followed by $39 \%$ of women with type II (removal of the clitoris and part or all of the labia minora), with $5 \%$ having undergone the most extreme type, type III or infibulation.

The age at which girls underwent this procedure varied. The mean age, among those who knew the age of FGC, was eight years old. Almost all had undergone the procedure before the age of 18 .

A number of different instruments were reported as being used during FGC, notably razor blades, knives, fiber from millet and stones. After circumcision, parents were said to take care of the girl by more than half of the respondents, and the cutters themselves for a third of the cases. Modern and traditional products are used both separately and 
combined to care for resulting wounds. These products include penicillin cream (63\%), alcohol (39\%), roots, (25\%), carité butter (10\%), and cow dung (2\%).

Among cut women, $14 \%$ had visible complications, especially keloids (62\%), stenosis $(20 \%)$ and vaginal obstructions (6\%). Among women who had undergone genital cutting, those with type III were the most likely to have an observable complication, and those with type I were the least likely.

The study also looked at client perspectives on FGC. The findings were generally positive: the majority of clients indicated that they are in favor of stopping the practice and do not want to have their daughters cut; the clients who were not cut want to remain that way. These findings vary by education level and FGC status. While these are encouraging results, they must be interpreted carefully, since the illegality of the practice in Burkina Faso may have biased clients' responses to these questions.

\section{Conclusion and recommendations}

This study took place in the socio-cultural context of rural Burkina Faso and provides information that can contribute to the fight against female genital cutting. The results were disseminated nationally, but, in order to conform with the philosophy of the LSC, they will need to be disseminated among local partners and community members as well.

These study findings need to be used during IEC workshops and interchanges both at the community and national levels. Given that the providers received training, they will need to be encouraged to integrate the fight against FGC into the daily activities of the SDPs included in this study. 


\section{INTRODUCTION A L'ETUDE}

\subsection{Contexte et identification du problème}

L'excision ou la circoncision féminine, couramment appelée ces dernières années mutilation génitale féminine (MGF), décrit un ensemble de pratiques qui consistent à une ablation partielle ou totale du clitoris et/ou des organes génitaux externes (petites et/ou grandes lèvres) de la femme, pratiquées pour des raisons culturelles ou autres et non à des fins thérapeutiques. Cette pratique, du fait des préjudices physiques, moraux, physiologiques et psychologiques qu'elle cause, constitue de nos jours un des problèmes de santé publique. En même temps, la pratique de l'excision pose un problème de droit de la personne humaine en général et de la femme en particulier. Elle est classée parmi les pratiques traditionnelles néfastes. Cette préoccupation a pris son essor depuis la Conférence Internationale sur la Population et le Développement (CIPD, 1994) au Caire, et cette volonté de lutter contre ce fléau a été réaffirmée à Beijing (1995).

L'Organisation Mondiale de la Santé (OMS) décrit quatre catégories d'excision :

Type I : Clitoridectomie

Type II : Clitoridectomie + ablation +/- totale des petites lèvres

Type III : Infibulation

Type IV : Autres opérations pratiquées sur les parties génitales externes, y compris l'introcision, la perforation ou l'incision du clitoris et/ou des petites lèvres, l'élongation du clitoris et/ou des lèvres, la cautérisation, le raclage et/ou l'incision du vagin, l'introduction de substances corrosives et d'aromates dans le vagin et de pratiques analogues.

Au Burkina Faso, un Comité National de Lutte Contre la Pratique de l'Excision (CNLPE), chargé de coordonner toutes les activités en matière de lutte contre la pratique de l'excision, a été mis en place depuis 1985. Entre autres activités, des associations et ONG nationales se sont engagées dans la lutte contre la pratique de l'excision. Le gouvernement Burkinabè a souscrit à cette lutte en adoptant depuis trois ans, une loi interdisant, sous peine de punition, la pratique.

Malgré ces efforts, la pratique persiste. Au niveau national, la prévalence est estimée à $70 \%$ selon les rapports du CNLPE. Dans la province du Bazèga, l'enquête de base menée dans le cadre des activités du Laboratoire de Santé Communautaire (LSC) a révélé une prévalence de $79 \%$; de même, $90 \%$ des hommes et des femmes interrogés approuvaient la pratique. Cependant, le type d'excision pratiquée n'est pas connu, de même que les complications éventuelles. C'est pourquoi l'étude sur la typologie de l'excision a été initiée. Cette étude a donc pour objectif d'évaluer la prévalence de la typologie et des complications liées à l'excision chez les patientes fréquentant les formations sanitaires du LSC. Les résultats de cette étude pourront également aider à comprendre les déterminants liés aux complications et à la pratique elle-même, ce qui est utile pour l'élaboration de stratégies appropriées en matière de lutte contre la pratique de l'excision. 


\subsection{Justification de l'étude}

Compte tenu des effets de cette pratique sur la santé des filles et des femmes, le Ministère de la Santé a identifié la recherche opérationnelle comme approche permettant de mieux comprendre et agir conséquemment contre la pratique en vue de contribuer à l'éradication des MGF par le biais de stratégies efficaces. La présente recherche opérationnelle, s'inscrit dans le cadre du Laboratoire de Santé Communautaire du Bazèga, une structure du Ministère de la Santé qui est chargée de tester des stratégies innovatrices en matière de santé de la reproduction avec la pleine participation de la communauté et en collaboration avec le Population Council dans le cadre du Projet de Recherche Opérationnelle et Assistance Technique en Afrique, phase II (RO/AT II).

Bien que l'excision soit prévalente dans le pays, aucune donnée n'existe sur le type d'excision le plus fréquemment rencontré et les complications y afférentes. De telles informations sont essentielles pour permettre au personnel de santé et aux gestionnaires des programmes de planifier des actions idoines en matière d'intervention et de prévention de la pratique, mais aussi des complications potentielles.

Cette étude a suivi, sur une période de trois mois, les femmes fréquentant les formations sanitaires du Bazèga et dont les motifs de consultation ont nécessité un examen du pelvis ; le statut d'excision et le type, de même que les complications médicales ont été observés et reportés. Les résultats de cette recherche vont servir de guide pour le développement de politiques et programmes sur les MGF au Burkina Faso et dans les autres pays du Sahel.

\subsection{Objectifs de l'étude}

\section{Objectif final :}

A long terme, cette étude contribuera à l'élaboration de programmes nationaux et régionaux visant à réduire les complications médicales causées par les MGF.

Les objectifs immédiats sont :

1. Décrire la prévalence et les types de MGF observées chez les femmes fréquentant les formations sanitaires de la région;

2. Identifier les complications liées aux MGF chez les femmes fréquentant les formations sanitaires. 


\subsection{Approche méthodologique}

II s'agit d'une étude clinimétrique ayant porté sur les clientes fréquentant les formations sanitaires (FS) et pour lesquelles un examen gynécologique était nécessaire. II s'agissait essentiellement de clientes venues consulter pour des soins prénatals, la planification familiale, des infections du tractus de la reproduction ou des soins gynécologiques et obstétricaux (y compris l'accouchement) ou pour tout autre motif nécessitant un examen gynécologique. Au début de la consultation, le consentement verbal des femmes a été demandé après une information claire sur l'objet de l'étude et les procédures de l'examen. De même, un anonymat a été respecté dans la collecte de l'information. Au total, 1920 clientes ont été recrutées autour des 21 FS de la zone du LSC (cf détail en annexe 1).

La mise en œuvre de cette étude a comporté plusieurs phases :

- l'élaboration des outils de l'étude et d'un manuel d'utilisation de l'enquêteur.

- la formation des prestataires chargés de l'étude à raison de deux par formation sanitaire ; il s'agissait des accoucheuses auxiliaires, des sages-femmes et des matrones, chargées des soins gynécologiques et obstétricaux, des infirmiers d'état ou brevetés. Au total 42 prestataires de services ont été formés en deux sessions (Kombissiri et Tanghin-Dassouri) de deux jours chacune. La formation a été assurée conjointement par la DSF, les médecins chefs des districts sanitaires de Kombissiri, Saponé et Manga avec l'appui du Docteur M. Akotionga, Gynécologue Obstétricien, formateur spécialiste des complications des MGF, du Centre Hospitalier Universitaire et membre du CNLPE. Cette formation a porté sur la définition du concept de MGF, la description des différents types de MGF et de leurs complications possibles, le protocole d'interrogatoire et de l'examen physique et gynécologique et également sur le remplissage de l'instrument de collecte des données.

- la collecte des données proprement dite suit une période de trois mois, de mai à juillet 1998.

- la supervision des prestations par les médecins chefs des districts sanitaires concernés (Kombissiri, Manga et Saponé) et le coordonnateur du LSC. Elle a permis non seulement de s'assurer du respect de la méthodologie et de régler d'éventuels problèmes logistiques mais également d'assurer l'assemblage des supports remplis.

- Les données ont été saisies sur Epi Info puis analysées sur le logiciel SPSS. L'analyse et l'interprétation ont été assurées par l'équipe de recherche avec l'assistance technique du Population Council. 


\section{RESULTATS}

\subsection{Motif de consultation}

Pour des questions de commodité, les motifs de consultations retenus ont été ceux pour lesquels un examen gynécologique était nécessaire en dehors même des objectifs visés par l'étude. Par motif gynécologique, il faut comprendre toute affection intéressant les organes génitaux externes et internes mais non liées à une grossesse. Les maladies sexuellement transmissibles (MST) ont bien été identifiées dans cette étude afin de connaître la part des MST dans les motifs de consultation. 57\% des clientes sont venues pour des consultations prénatales (CPN), $12 \%$ pour la planification familiale (PF) et $12 \%$ pour les accouchements. Les consultations gynécologiques et les MST sont respectivement de $7 \%$ et $3 \%$.

\subsection{Les caractéristiques socio-démographiques}

Cette partie caractérise le profil de la cliente ; les questions entrent dans le cadre global de l'anamnèse médicale. Le tableau 1 résume les caractéristiques sociodémographiques et montre les similarités et les différences de ces caractéristiques entre les femmes excisées (93\%) et les femmes non-excisées (7\%).

Tableau 1: Caractéristiques socio-démographiques et statut d'excision des clientes interviewées

\begin{tabular}{|c|c|c|c|}
\hline Caractéristiques & Clientes non-excisées & Clientes excisées & Toutes les clientes \\
\hline $\mathrm{Age}^{\star \star}$ & $(n=128)$ & $(n=1764)$ & $(n=1892)$ \\
\hline $15-20$ ans & 38 & 23 & 24 \\
\hline $21-25$ & 33 & 26 & 27 \\
\hline $26-30$ & 16 & 24 & 23 \\
\hline $31-35$ & 8 & 14 & 13 \\
\hline $36-40$ & 5 & 9 & 9 \\
\hline $41-45$ & 0 & 3 & 3 \\
\hline 46 et plus & 0 & 1 & 1 \\
\hline Niveau de scolarisation ${ }^{\star \star}$ & $(\mathrm{n}=129)$ & $(n=1781)$ & $(n=1910)$ \\
\hline Pas de niveau d'instruction & 59 & 79 & 77 \\
\hline Alphabétisé & 6 & 8 & 8 \\
\hline Primaire & 24 & 10 & 11 \\
\hline Secondaire & 11 & 3 & 4 \\
\hline Ethnie $^{\star \star}$ & $(\mathrm{n}=129)$ & $(n=1789)$ & $(n=1918)$ \\
\hline Mossi & 87 & 95 & 95 \\
\hline Peulh & 5 & 4 & 4 \\
\hline Autres & 8 & 1 & 1 \\
\hline Religion $^{\star \star}$ & $(n=130)$ & $(n=1790)$ & $(n=1920)$ \\
\hline Musulmane & 27 & 48 & 46 \\
\hline Catholique & 48 & 39 & 40 \\
\hline Animiste & 8 & 8 & 8 \\
\hline Protestante & 17 & 5 & 6 \\
\hline Statut matrimonial $^{\star \star}$ & $(n=130)$ & $(n=1788)$ & $(n=1918)$ \\
\hline Monogame & 54 & 44 & 45 \\
\hline Polygame & 28 & 46 & 44 \\
\hline Concubinage & 8 & 6 & 6 \\
\hline Célibataire & 9 & 3 & 4 \\
\hline Veuve/Divorcée & 1 & 1 & 1 \\
\hline
\end{tabular}

${ }^{* *}$ Test $x^{2}$ entre excisées et non-excisées $p<.001$ 
Age de la cliente : En zone rurale, il est parfois difficile d'obtenir l'âge exact de la cliente. L'âge des patientes se situe entre 15 et 55 ans, mais la plupart d'entre elles ont entre 15 et 30 ans. C'est une population relativement jeune, surtout parmi les femmes non-excisées: $87 \%$ des femmes non-excisées ont entre 15 et 30 ans, contre $73 \%$ des femmes excisées $(p<.001)$.

Situation matrimoniale : En ce qui concerne la situation matrimoniale, la plupart des patientes étaient mariées ( $89 \%$ des cas) avec une proportion de $45 \%$ de monogames et $44 \%$ de polygames. $54 \%$ des femmes non-excisées étaient monogames contre $28 \%$ polygames. Les célibataires représentaient seulement $4 \%$ de l'échantillon, avec $1 \%$ de femmes veuves ou divorcées.

Ethnie: Etant donné que l'étude se déroule dans une zone où l'ethnie Mossi est prédominante, il n'est pas étonnant que celle-ci représente $95 \%$ de l'ensemble des 1920 clientes recrutées.

Niveau d'instruction : La majorité des patientes interviewées n'est pas instruite, 77\% des cas. Seulement $11 \%$ ont un niveau primaire et $4 \%$ un niveau secondaire. Huit pour cent $(8 \%)$ ont reçu une alphabétisation fonctionnelle en langue locale. On constate une différence entre les femmes excisées et les autres: les femmes non-excisées sont plus éduquées, $35 \%$ d'elles ont un niveau primaire ou secondaire, contre $13 \%$ des femmes excisées $(p<.001)$. Lorsqu'on effectue une régression logistique en incluant toutes ces caractéristiques socio-démographiques, le niveau d'éducation de la femme et le statut d'excision apparaîssent liés : plus la femme est éduquée, moins elle court le risque d'être excisée $(p<0.05)$.

Religion : Les religions musulmane et catholique sont prédominantes. Les animistes et les protestants représentent $8 \%$ et $6 \%$ respectivement.

\subsection{Statut obstétrical}

Quatre vingt deux pour cent (82\%) des femmes avaient déjà eu au moins un accouchement. Parmi ces femmes, l'âge au premier accouchement varie de 12 à 31 ans avec une moyenne de 19 ans. Cette donnée est superposable aux résultats de l'Enquête Démographique et de Santé (EDS). L'âge moyen au mariage dans le milieu Mossi survient autour de 17 ans. De même nous notons une forte natalité au sein de ce groupe, le nombre variant de 0 à 12 avec une moyenne de 3 accouchements par femme.

\subsection{Expérience des clientes en matière d'excision}

\subsubsection{Statut d'excision et type}

L'excision reste une pratique encore très répandue ; en effet, les résultats révèlent que sur les 1920 enquêtées, 1790 étaient excisées, soit 93\%. Parmi les femmes excisées, trois des quatre types d'excision ont été observés (selon la définition de l'OMS). 
Parmi les patientes excisées, $56 \%$ avaient subi le type I, tandis que $39 \%$ et $5 \%$ avaient été victimes respectivement des types II et III (graphique 1). Ces taux sont superposables aux tendances mondiales. En effet, on estime à $85 \%$ la prévalence des types I et II exceptés dans les pays de la corne de l'Afrique (Djibouti, Somalie, Soudan) où le type III représente 80 à $90 \%$ de Graphique 1: Statut d'excision des clientes et types d'excision observés

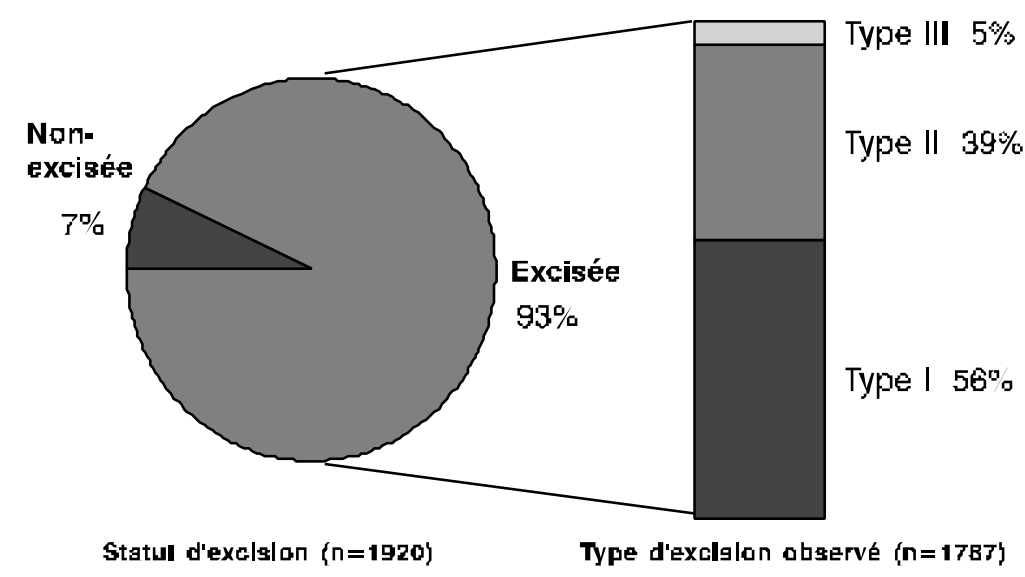
toutes les MGF.

\subsubsection{Age à l'excision}

Dans le passé, l'excision était pratiquée à un âge avancé dans la mesure où elle constituait un rite initiatique du passage de l'adolescence à l'âge adulte. De nos jours force est de constater que l'excision se pratique à des âges beaucoup plus jeunes, avant la puberté comme l'indique les résultats de cette étude (âge moyen à l'excision = 8 ans) et bien d'autres ; $18 \%$ des femmes sont excisées avant l'âge de 5 ans. Une partie non moins importante, $38 \%$ ne connaissaient pas leur âge à la date de l'excision, ce qui laisse croire qu'elles furent excisées à un âge où elles n'étaient pas en mesure de s'en rappeler. Dans tous les cas, la quasi totalité des excisées le sont avant l'âge de 18 ans (graphique 2), qui est l'âge présumé de

Graphique 2 : Probabilité cumulative d'être excisée par âge

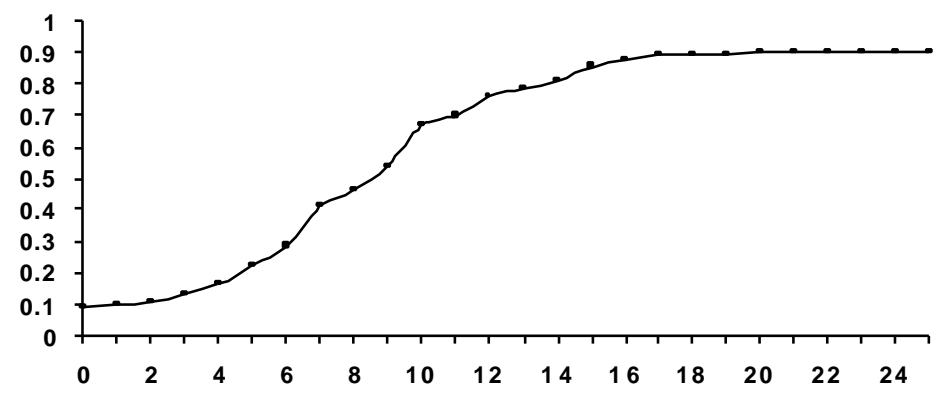

L'âge médian de survie $=9.47$ ans, $n=1239$ mariage dans la culture du milieu.

\subsubsection{Instrument utilisé pour l'excision}

Plusieurs instruments sont utilisés pour pratiquer l'excision ; il peut s'agir d'un couteau, d'une lame, de pierre taillée ou de brindille de tige de mil. Parmi les femmes qui savaient quel instrument avait été utilisé, $68 \%$ des cas ont cité la lame, suivie du 
couteau dans $29 \%$ des cas. D'autres instruments comme les pierres taillées et les fibres de tiges de mil ont été mentionnés par $3 \%$ des femmes.

La notion d'instrument utilisé est très importante dans la mesure où ils peuvent être sources ou vecteurs des infections faisant suite à l'excision. Par ailleurs, un même instrument peut être utilisé pour exciser plusieures femmes, souvent sans désinfection entre les interventions. Cette pratique est considérée comme étant une source potentielle de contamination pour d'autres maladies comme l'hépatite ou le SIDA.

\subsubsection{Personnes ayant assuré les soins}

Parfois, après l'excision, plusieurs cas de figures peuvent se présenter quant aux soins de la plaie de l'excisée. Ce peut être l'exciseuse qui en assure les soins, ou encore les parents, en l'occurrence la mère, une tante ou une tierce personne du village. Pour ce qui est de notre échantillon, les soins avaient été assurés surtout par les parents dans la moitié des cas (52\%) tandis que l'exciseuse elle-même était intervenue dans $1 / 3$ des cas (graphique 3). De plus, le personnel de santé peut être impliqué dans le traitement, soit sciemment ou au cours d'une complication (hémorragie, infections, rétention d'urines...). C'est ainsi que dans cette étude, le personnel de santé est intervenu dans le cadre des soins d'une plaie d'excision dans un pour cent $(1 \%)$ des cas.

II a souvent été rapporté des cas d'infections à germes banaux ou parfois des cas de tétanos suite à une excision; aussi, l'une des questions de cette étude était de connaître les produits utilisés pour les soins des plaies. Plusieurs produits traditionnels ou modernes supposés être dotés de pouvoir cicatrisant avaient été utilisés ; il s'agit des produits pharmaceutiques modernes tels que l'alcool $(39 \%)$ et la pommade pénicilline $(63 \%)$; il a été mentionné l'usage des racines, de la bouse de vache et du beurre de karité dans respectivement $25 \%$, $2 \%$ et $10 \%$ des cas. Ces produits sont la plupart du temps utilisés en combinaison.

\subsection{Complications observées}

On reconnaît à la pratique de l'excision un certain nombre de complications et conséquences; en effet, elle porte atteinte à l'intégrité physique et psychique de la femme et peut être vectrice de complications d'ordre médical, physique, psychologique pouvant entraver sa vie obstétricale future. Ces complications sont soit immédiates soit à long terme. 
Parmi les femmes excisées, $14 \%$ de complications

diverses-physiques

et/ou apparentes-ont été observées

(graphique 4), l'étude n'a pas porté sur d'autres complications, telles que des complications psychologiques ou des complications qui auraient été guéries dans le temps. Les chéloïdes et les sténoses étaient les complications les plus fréquemment

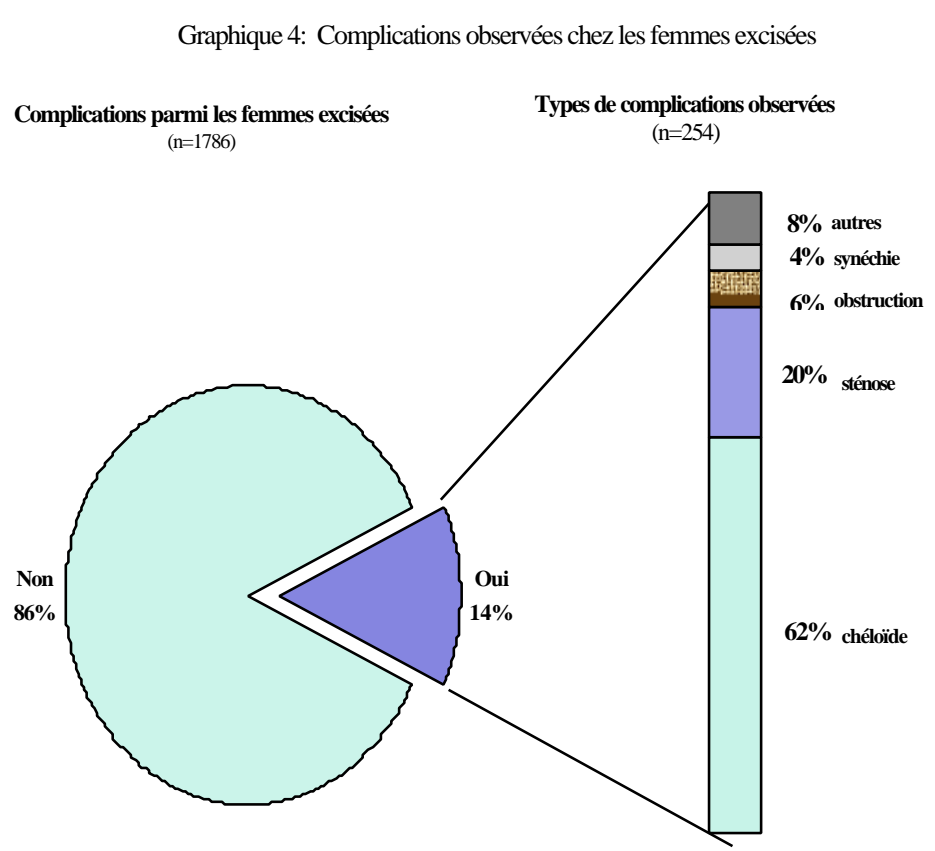

rencontrées, respectivement dans $62 \%$ et $20 \%$ des cas. Les synéchies vaginales et les obstructions - rétrécissement constituent $10 \%$ des cas observés. En faisant une régression multiple avec les facteurs socio-démographiques et le nombre d'accouchements, les femmes excisées du type III se sont révélées être les plus propices à avoir des complications observables lors de l'examen, suivi par le type II ; les femmes du type I étant les moins susceptibles d'avoir ces types de complications $(p<.001)$.

\subsection{Signes d'infection génitale}

L'examen d'une patiente est une opportunité pour dépister et traiter toute autre affection même non liée au motif de consultation. Aussi la pertinence de la recherche des signes d'infection génitales se situe dans cette logique. On voudrait savoir si la femme a des symptômes évoquant une MST. Si tel est le cas, un traitement ou une référence pour traitement a été systématique. Les données ont révélé que $24 \%$ des clientes montraient un ou plusieurs symptômes évoquant une MST. II s'agissait essentiellement d'écoulements anormaux (19\%), de signes d'irritation (4\%), d'ulcération (2\%), et de végétation (1\%).

La comparaison entre le statut d'excision et les signes d'infection génitale a montré que les femmes excisées étaient 1,6 fois plus susceptibles d'avoir de tels signes que les femmes non excisées, sans une différence significative entre les types d'excision. Les femmes excisées avec complications apparentes telle une chéloïde étaient beaucoup plus susceptibles d'avoir des signes d'infection que les femmes excisées sans complications $(p<.001)$.

\subsection{Difficultés à l'accouchement}

$51 \%$ de clientes qui avaient accouché une ou plusieures fois ont rapporté avoir eu des difficultés avec un accouchement : $53 \%$ de femmes étaient excisées contre $27 \%$ de 
femmes non excisées. Les femmes excisées-en tenant compte des caractéristiques socio-démographiques-étaient 3 fois plus susceptibles de rapporter des difficultés à l'accouchement. L'âge de la femme était l'autre variable significative, les jeunes femmes étant plus enclines aux difficultés. Le même type d'analyse a montré aussi que les femmes excisées du type III ont reporté le plus de telles difficultés.

\subsection{Perspectives et attitudes des femmes en matière d'excision}

\subsubsection{Excision de la femme dans le futur}

L'étude a aussi exploré le risque que couraient les femmes non encore excisées de l'être. Parmi les 128 non excisées de l'échantillon, 8 d'entre elles (entre 18 et 30 ans), soit $6 \%$ affirment qu'elles seront excisées dans l'avenir tandis que $94 \%$ souhaitent ne pas l'être. Ces chiffres laissent percevoir une possibilité de réduction de la pratique. Toutefois, ces chiffres doivent être aussi analysés en tenant compte du contexte, à savoir que l'excision est punie par la loi au Burkina Faso.

\subsubsection{Disposition des femmes à faire exciser leur fille à l'avenir}

Un nombre important de clientes, $71 \%$, affirment qu'elles ne feront pas exciser leur fille. Douze pour cent $(12 \%)$ se sont abstenues de toute réponse, préférant garder le silence ; cela serait-il lié à l'impact de la loi ou au centre de décision qui revient à l'homme ou aux grands parents ? En réalité, l'organisation des familles africaines rend complexe le circuit de la décision surtout pour des aspects à connotation socioculturelle.

Le fait de vouloir ou non exciser sa fille à l'avenir varie en fonction du statut d'excisée ou non de la cliente ; en effet, les femmes excisées courent plus de risque d'exciser leur filles que les femmes non excisées (graphique 5).

Cette partie cherche à savoir les raisons de la bonne pratique, c'est-àdire celles qui encourageraient les femmes à ne pas exciser leurs filles.

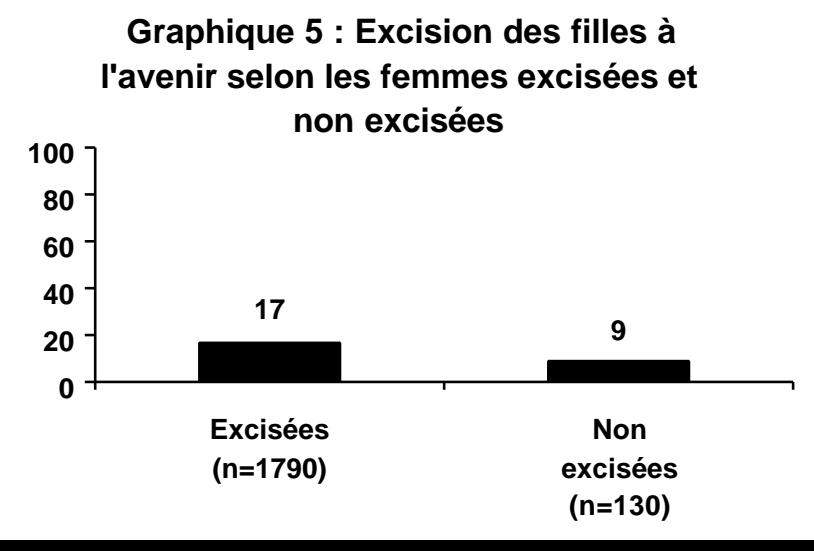
Celles qui sont souvent évoquées sont relatives aux messages de sensibilisation notamment l'interdiction de la pratique par la loi, les complications subséquentes et surtout les difficultés à l'accouchement, etc.

\subsubsection{Disposition des femmes à l'arrêt de l'excision}

Cette section voudrait renseigner si les femmes sont favorables à l'arrêt de la pratique de l'excision. La situation est favorable à l'arrêt de l'excision. Cependant nous devrons prendre en compte le fait que l'interview se déroule dans une formation sanitaire et aussi que l'excision est interdite au Burkina Faso. Des 1679 répondantes, 1426 soit $74 \%$ sont favorables à l'arrêt de l'excision; ce qui constitue un progrès par rapport aux attitudes pro excision estimée à environ $50 \%$ en 1995 (Analyse situationnelle du programme de planification familiale au Burkina Faso, 1995). II n'existe pas de 
différence significative en fonction du statut d'excision de la femme comme l'indique le graphique 6 .

\section{Dissémination et utilisation}

\subsection{Dissémination}

Elle est envisagée à trois niveaux dont deux ont déjà été accomplis:

- Au niveau national, les résultats ont été présentés lors des journées de dissémination des résultats du Laboratoire de Santé Communautaire qui ont eu Graphique 6 : Répartition des femmes excisées et non excisées favorables à l'arrêt de l'excision

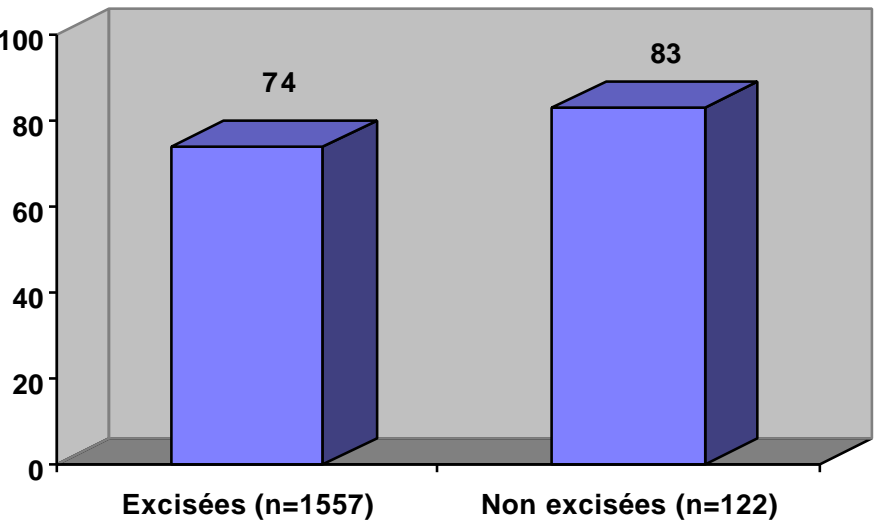
lieu du 15 au 16 décembre 1998 à Ouagadougou.

- Au niveau régional et international, les résultats partiels ont été présentés à la conférence de fin de projet à Dakar, Sénégal, en septembre 1998 et à la réunion annuelle de " Population Association of America » ; l'étude fait partie également de la liste électronique de Population Council.

- Au niveau local, une rencontre de restitution des résultats d'une demi-journée organisée à Kombissiri est prévue et regroupera tout le personnel médical ayant participé à l'étude, et les membres de l'équipe cadre des districts de Saponé et Tanghin-Dassouri, y compris les sections locales du CNLPE ; ceci aurait pour effet d'encourager le personnel des formations sanitaires à réfléchir sur les moyens d'introduire des messages anti-MGF dans leurs formations sanitaires à partir de ces informations.

- Au niveau communautaire, les comités de gestion de la zone d'intervention devraient prendre connaissance des résultats, ce qui permettrait d'obtenir leur participation dans les actions en matière de lutte contre l'excision qui seront entreprises ultérieurement.

\subsection{Utilisation des résultats}

Les données collectées seront très utiles pour faire avancer les efforts nationaux entrepris pour la lutte contre les MGF et pour l'élaboration de stratégies de prise en charge des complications médicales des MGF, par la sensibilisation des prestataires de services comme des membres de la communauté sur les conséquences des MGF.

Une étude participative sur la pratique de l'excision est déjà menée au Bazèga et où la communauté a proposé des axes d'intervention incluant l'IEC ; les résultats de cette étude pourront être utilisés pour la mise en œuvre de ces interventions. 


\section{RESUME DE RESULTATS CLES}

- Sur un échantillon de 1920 clientes, 97\% d'entre elles étaient excisées.

- La moyenne d'âge à l'excision est de 8 ans.

- Les types d'excision observés sont de $56 \%$ pour le type I, 39\% pour le type II et $5 \%$ pour le type III.

- Parmi les femmes excisées, $14 \%$ d'entre elles ont connu une complication observable liée à l'excision dont $62 \%$ de chéloïdes et $20 \%$ de cas sténoses et d'obstruction vaginale.

- Statiquement les femmes ayant subi l'excision du type III sont plus enclins à avoir des complications observables.

- Il y a un lien statistiquement significative entre le statut d'excision et la possibilité d'avoir une difficulté à l'accouchement. Les femmes excisées étaient 3 fois plus susceptibles de rapporter des difficultés à l'accouchement.

- De même les clientes excisées du type III sont significativement plus nombreuses à rapporter des complications à l'accouchement

- Plus la femme est éduquée, moins elle court le risque d'être excisée; cette différence est significative.

- Les femmes excisées avec des complications apparentes étaient plus susceptibles d'avoir des signes d'infection génitale.

Les femmes excisées courent plus de risque d'exciser leurs filles à l'avenir que les non excisées.

- II n'y a pas de différence significative entre les femmes excisées et non excisées pour ce qui concerne leur approbation à l'arrêt de l'excision. 


\section{- IV. CONCLUSION ET RECOMMANDATIONS}

\section{Conclusion 1}

Cette étude est une première en zone rurale ; dans le passé, la typologie de l'excision et les complications afférentes n'ont pas fait l'objet d'étude conséquente. Bien que limitée à une zone socio-culturelle du Burkina, l'étude donne des indications et des données qui peuvent contribuer à la lutte contre la pratique de l'excision.

\section{Recommandation 1}

Les résultats de cette étude doivent être largement diffusés aux institutions, ONGs et Associations intervenant en matière de lutte contre l'excision dans le but de les encourager à les utiliser dans les campagnes d'IEC à l'intention des publiques cibles.

\section{Conclusion 2}

Une étude participative sur la pratique de l'excision menée dans la zone du LSC a abouti à des propositions d'actions à mener au niveau communautaire, dont la démonstration sur les méfaits de l'excision sur la santé de la femme.

\section{Recommandation 2}

La restitution des principaux résultats à la communauté pourrait aider à la mise en œuvre des programmes en matière de lutte dans la zone du LSC. Ces résultats peuvent aider efficacement à la mise en œuvre des recommandations de l'étude participative.

\section{Conclusion 3}

La lutte contre l'excision devrait se mener au niveau communautaire mais aussi des formations sanitaires. Cette étude a connu l'adhésion et la participation des prestataires cliniques qui ont reçu une formation en matière d'excision et dans la collecte des données.

\section{Recommandation 3}

Etant donné que les prestataires ont reçu une formation, il serait utile et pertinent d'intégrer la lutte contre l'excision dans les prestations usuelles des formations sanitaires.

\section{Conclusion 4}

Dans la plupart des études, des pathologies liées à la non excision des filles ont fréquemment été évoquées par la communauté. La présente étude n'a pas abordé ces aspects.

\section{Recommandation 4}

II serait souhaitable de mener une étude dans le but d'identifier la fréquence de telles pathologies au sein des communautés où l'excision n'est pas pratiquée, ce qui serait également un plus dans le cadre de la lutte contre la pratique. 


\section{ANNEXES}


ANNEXE 1. Echantillonnage des formations sanitaires et des enquêtées par zone.

\begin{tabular}{|c|c|c|}
\hline ZONE & $\begin{array}{l}\text { FORMATION } \\
\text { SANITAIRE }\end{array}$ & $\begin{array}{c}\text { NOMBRE } \\
\text { ENQUETEES }\end{array}$ \\
\hline $\mathbf{A}$ & $\begin{array}{l}\text { CM Kombissiri } \\
\text { Toudou } \\
\text { Toécé } \\
\text { Bonsrima } \\
\text { Zangogho } \\
\text { Gaongho } \quad \text { Sous total A }\end{array}$ & $\begin{array}{r}114 \\
79 \\
110 \\
103 \\
80 \\
38 \\
\mathbf{5 2 4} \\
\end{array}$ \\
\hline B & $\begin{array}{l}\text { Tanghin Dassouri } \\
\text { Bassemyam } \\
\text { Komsilga } \\
\text { Lugsi } \\
\text { Tintilou Nord } \\
\text { Tintilou Sud (Konki Ipala) } \\
\text { Vipalogho } \\
\quad \text { Sous total B }\end{array}$ & $\begin{array}{r}187 \\
48 \\
58 \\
64 \\
13 \\
60 \\
96 \\
\mathbf{5 2 6}\end{array}$ \\
\hline $\mathbf{C}$ & $\begin{array}{l}\text { Béré } \\
\text { Bindé } \quad \text { Sous total C }\end{array}$ & $\begin{array}{r}122 \\
140 \\
\mathbf{2 6 2}\end{array}$ \\
\hline D & $\begin{array}{l}\text { CM Saponé K. } \\
\text { Saponé Marché } \\
\text { Sanbin } \\
\text { Rakaye } \\
\text { Ipelcé } \\
\text { Doulougou } \\
\end{array}$ & $\begin{array}{r}222 \\
81 \\
83 \\
67 \\
60 \\
95 \\
\mathbf{6 0 8}\end{array}$ \\
\hline TOTAL & & 1920 \\
\hline
\end{tabular}

